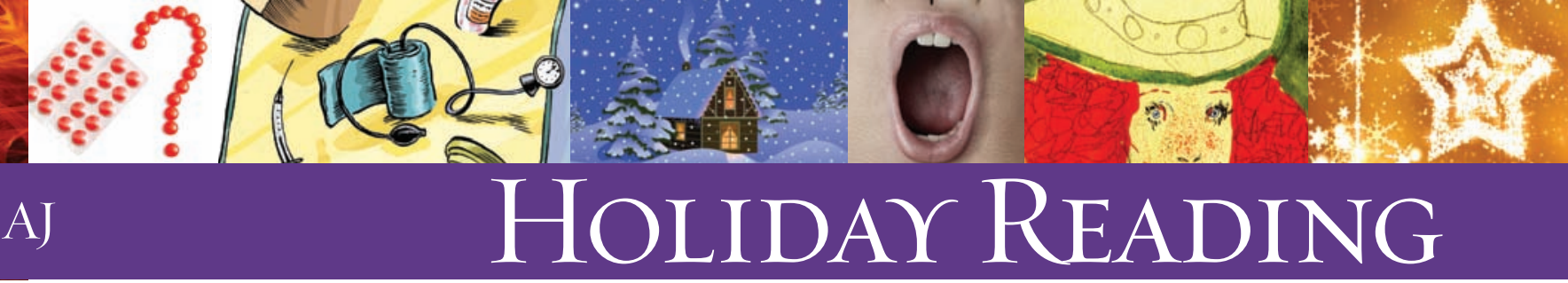

SATIRE

\title{
The anemic maternal proxy and the seven resident stakeholders: a case for knowledge translation
}

\section{Sheila Strong}

\section{The case}

A female youth presented with anemic pallor. She reported having resided in a monarchically structured environment with a pre-established stratification hierarchy based entirely on human facial aesthetics. When her attainment of adolescence compromised the prevailing hierarchic order, the result had been a top-down restructuring plan with homicidal end-points.

Lacking access to Knowledge Diplomacy Intervention resources, the youth had elected to effect a simplified exit strategy. With assistance from the Research Group for New Important-Sounding Paradigms (RGNISP), she eventually identified an overcrowded residential structure situated in a rural undeveloped forested environment as the target for an impromptu accommodation agreement.

The structure housed seven diminutive proprietors who had, respectively:

- a mood disorder

- narcolepsy

- generalized social anxiety

- endogenously inhibited serotonin reuptake

- an academic doctorate

- an unidentified allergen sensitivity

- generalized developmental delay

\section{Knowledge model}

Using process guidelines conceived, developed, owned, repeatedly cited and extensively promoted by the Acronyms and Big Words Think-Tank Centre (www.BigFluffy Words.org), a proposal was drafted whereby the anemic female youth would fulfill a maternal proxy role for the seven resident stakeholders. The proposal was actioned in alignment with the following agreed-upon end-goals:

- to effect a sustained increase in safety benefit for the anemic female youth by leveraging the iterative security inherent in a group-based system;

- to increase uptake of home organization systems by the seven resident stakeholders - or, more simply, to positively alter the negative sanitation variables inherent in an overcrowded familial accommodation domain.

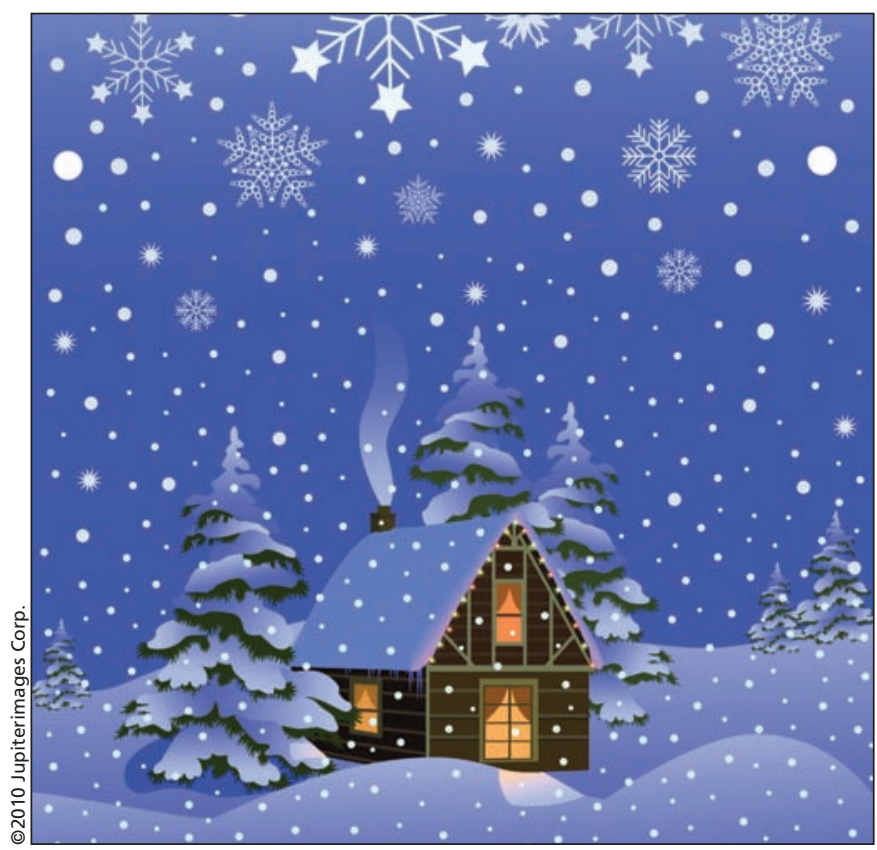

\section{End-outcome}

After a brief time-based interval, the primary political opponent of the female youth achieved a verified ascertainment of identification of the youth's geographic domain. A targeted Vulnerability Creation Action Cycle was initiated. The implementation phase of this knowledge-based cycle ultimately resulted in the incapacitation of the youth and an end to the familial proxy agreement in practice.

Emergency knowledge assistance was quickly dispatched in the form of a field group of researchers from RGNISP, who elaborated a chart extraction grid.

\section{What this means in terms of implications}

Much can be learned from the breakdown of this model. First, it should be noted that any knowledge-based system is

Sheila Strong is a manuscript editor for CMAJ. 


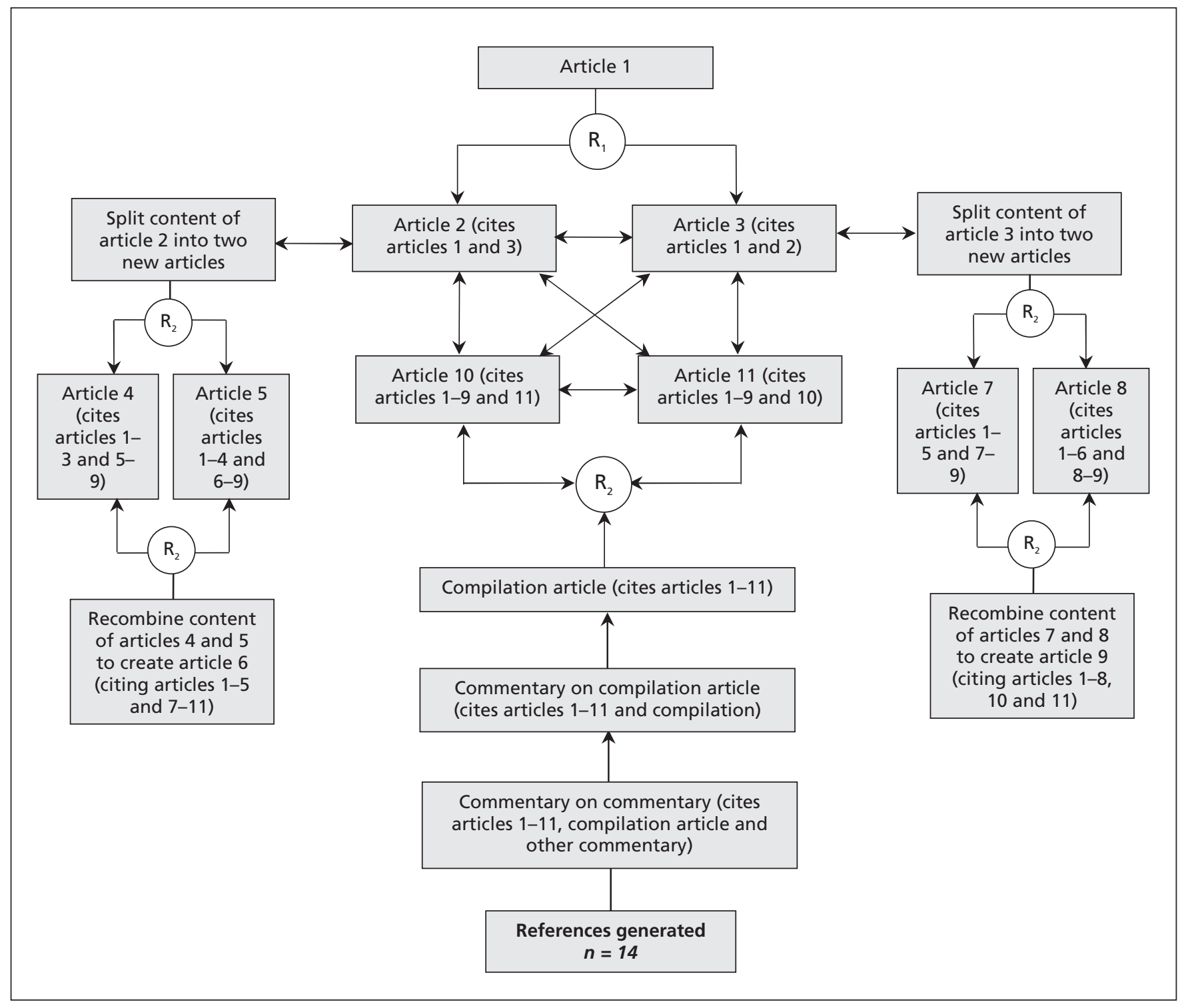

Figure 1: The Reflexive Referencing Self-Perpetuation Model. Note: R1 = begin rotating order of coauthor names in bylines. $\mathrm{R} 2$ = recycle content.

only as morally and ethically sound as its individual enduser. Open access to knowledge-based model development tools by homicidal individuals is a limitation inherent in tools of this nature.

A three-part assessment analysis has been actioned by the Variable Appraisal Consortium for Actualizing kNowledge Translation (VACANT).* Its objectives are two-fold:

- to document the mechanisms leading to failure of the proxy agreement between the youth and the seven resident stakeholders;

\footnotetext{
*Members of VACANT comprise all of the members of the Acronyms and Big Words Think-Tank Centre and RGNISP, plus LZ (to whom we promised authorship status in return for catering), and possibly AA (who is an old colleague and likes to be included in these kinds of things) and GY (who always uses big words that sound incredibly cool).
}

- to build onto the growing list of references used by the group to cite its own work (see the Reflexive Referencing Self-Perpetuation Model, shown in Figure 1).

\section{Knowledge gaps}

Updated guidelines are needed for group-based security scenarios involving familial proxy agreements with pre-existing political variables. The Acronyms and Big Words ThinkTank Centre feels confident that VACANT will competently address this need with rigorous and systematic multicomponent needs assessment protocols, and, further, that these protocols will quickly be adopted as the recommended standard for facilitating guideline development.

The role of anemic morbidity in Vulnerability Action Cycle outcomes should also be examined. 